\title{
Community and health care involvement to controlling and Management of dengue fever in Pakistan
}

\author{
Tahmina Bano \\ Master of sciences in nursing from the University of Lahore \\ Muhammad Hussain \\ Associate professor Lahore School of nursing, the University of Lahore \\ Muhammad Afzal \\ Head of department Lahore school of nursing, the University of Lahore
}

\begin{abstract}
In recent years, dengue has become a shared infection transmitted by mosquitoes, one of the main international health problems. In broad spectrum clinical manifestations, symptomatic dengue virus can occur from gentle febrile sickness to hazardous shock disorder. As a contribution to the manifestation of the disease, both the viral factor and the host have an important role in each infection. It is significant to realize the burden of mortality and morbidity in health care. Early intuition and dengue fever diagnosis in primary health care services can minimize complications if managed appropriately. We must apprehend the intensity of the complication in in relation to clinical picture, treatment, transmission, diagnosis and prevention.
\end{abstract}

Keywords: Dengue, clinical features, pathogenesis, diagnosis, treatment.

DOI: $10.7176 / \mathrm{JMPB} / 62-07$

Publication date: December $31^{\text {st }} 2019$

\section{INTRODUCTION}

Dengue fever is commonly a mosquito bite disease and is a main health issue in subtropical and tropical regions of the world. Benjamin Rush first define the word dengue in 1789, a Spanish word that means "fastidious", derivative of the word "dinga", which means the break bone fever and the evil of the soul. The Flavivirus and Flaviviridae family of mosquitos are the causative agent of dengue fever. An infected female mosquito transmits the dengue virus in humans which belong to genus Aedes. Dengue fever represents an extensive series of clinical manifestations, comprising mild fever to severe forms (Keating J. 2001, 1587-97). Dengue Shock Syndrome (DSS) and Hemorrhagic Dengue (DHF) are the worsening form of Dengue. The common clinical symptoms of dengue fever are severe headache, vomiting, joint and muscle pain, nausea and rash. High fever and hemorrhagic phenomena are the features of DHF. Dengue is the foremost reason of hospitalization, and severe dengue fever manifests about 500,000 people which need hospitalization, dominated by children, with about $2.5 \%$ of affected people dying annually.

In the world; the most usual infection transmitted by mosquitoes is Dengue virus. These infections may or may not be dominant, and may also cause dengue hemorrhagic fever, undifferentiated fever, dengue fever (DF) or dengue shock syndrome (DHF / DSS). Four related but antigenically identified viruses are DEN 1, 2, 3 and 4 which causes DF. Dengue fever is an acute febrile condition. Aedes mosquitoes are the most important vector of the virus in humans. This virus affects the population in a wide spectrum and causes a very high mortality rate.

In tropical and subtropical regions of more than 100 countries, approximately 3 billion people are more likely to develop DV infection. Some common clinical features of patients suffering from DV infection are; lymphoma, leukopenia, lymphocytosis, high hematocrit and thrombocytopenia. Different serotypes due to secondary infections are responsible for primary infections and are considered as major elements in the epidemiology of DH / DSS. Early detection of dengue fever requires a more accurate and cost-effective diagnostic test. Many are practically available for dengue infection (Zhang B, Salieb-Beugelaar GB, Nigo MM, Weidmann M, Hunziker P.2015, 1745-61.) (Guzman MG, Kouri G. J ClinVirol 2003; 1-13). Delayed diagnosis and failure to detect impediments may lead to worse results.

\section{Local Prevalence}

In Pakistan, first dengue outbreak was reported in 1994. The outbreak in India and Sri Lanka was related with many serotypes of dengue virus, but DEN-3 was dominant and was different genetically from DEN-3 viruses which was isolated previously from people who were infected in these kingdoms. (Kumar K, Singh PK, Tomar J, Baijal S. Med 2010; 997-1000).Dengue virus is native to Pakistan, spreading throughout the year with maximum 
spread after monsoon. The recent floods in Pakistan have exacerbated the situation. Pakistan, 1809 indefinite dengue 881 approved by October 11, 2010, 5 dead cases reported, 15 were found to be deadly in Islamabad turned out to be confirmed. In primary health care units, mild to moderate fever is considered assumption of dengue. Investigators have determined that the co-circulation of DEN-2 and DEN-3 is liable for the outbreak in 2006 in Karachi. In both groups; Primary and secondary cases were identified. DHF cases exhibit a marginal relation with DEN-2. The new serotype introduction (DEN-3) and / or a genotypic change of the endemic serotype (DEN-2) are possible factors for the outbreak of DHF in this section (Gibbons RV, Vaughn DW. 2002; 1563-6).

\section{Clinical features of DF}

Most of the presented clinical cases are asymptomatic, with the disease starting from a mild flu to severe conditions such as vascular leakage, bleeding and even shock. DF is also known as febrile disease. Half of the patients have skin problems, while the fever stays between 5 and 7 days. A common symptom that appeared during the first 24-48 hours was flushing of face. Maculopapular or petechial rash occurs in patients during suspension (WHO. Managing Regional 2013; RPT125191-2.). Clinical symptoms included fever, headache, leukocytopenia, pain and rash in the muscles and bones. Because of obdurate pain, DF is also called as break bone fever. Pal et al discovered a significant relationship between dengue virus burden (VL) and interferongamma level (IFN-g). They determined that there is a strong association between fever, nausea, rash and pain, aches, leukopenia and persistent vomiting (Ooi EE, Goh KT, Gubler DJ. 2006; 12: 887-92).

\section{Pathophysiology}

Dengue fever arises from 4 antigenically dissimilar dengue virus serotypes: DENV-1, 2, 3 and DENV-4. Four types of viruses can cause serious diseases. These include West Nile virus, yellow fever virus, Louis Flaviviridae / family including the encephalitis virus and Japanese encephalitis virus are members of the genus Flaviviridae (Rigau-Pérez JG, Clark GG, Gubler DJ, Reiter P, Sanders EJ, Vorndam AV. 1998; 971-7). A. aegypti is the primary vector responsible for infection spreading, extremely domesticated mosquito bitten during the day, and albopictus is responsible for infection. Although mosquitoes are of Asian origin, they are currently reproduced in Africa, Europe and America. Both the vector and the virus have been transmitted through international travel and freight transport (Wasay M, Channa R, Jumani M, Zafar A. 2008; 488-9). Virion consists of a lipoprotein envelope, 3 structural proteins and 7 non-structural proteins in which the non-structural protein 1 (NS1) has pathological and diagnostic significance. With any type of serotype infection delivers long-term immunity to this specific serotype; however, cross-protection with other serotypes persists solitary for some months [Siddiqui FJ, Haider SR, Bhutta ZA. 2009; 3: 306-12). Some analysis have revealed that with the DENV-1 or DENV-2 serotype infection may lead to a much serious infection. Pathogenesis is related to the host's immune response. [Rasheed SB, Butlin RK, Boots M. 2013; 127:11-7] Primary infection is mostly benign. However, secondary infection with multiple infections or different serotypes may cause a serious infection which may be categorized as dengue shock syndrome or dengue hemorrhagic fever according to clinical findings. Dendritic cells have a part in the pathogenesis of antigen presenting with cell-mediated immune response and humoral immune response (Khan E, Hasan R. 2011; 26: 1-). The memory T cells proliferation and pro-inflammatory cytokines production lead to dysfunction of vascular endothelial cells, leading to plasma leakage.

\section{Primary prevention}

The World Health Organization commends approaches for the control and prevention of dengue infection, and establishments in the endemic areas of dengue can also produce their own initiatives and prevention programs. Communities in dengue endemic areas should be trained to recognize signs and inhibit transmission (Ashley EA. 2011; 1: 39-41).

\section{Secondary prevention}

The different serotypes may leading to secondary infections. After recovery from the initial infection, the standard primary prevention measures must be taken.

\section{Management}

Treatment is absolutely supportive, such as keeping the body temperature below $39^{\circ} \mathrm{C}$ and giving the patient paracetamol (up to four times per 24 hours). Patients are advised to avoid aspirin or Brufen / Ponston. It recommends drinking liquids containing water, soup, milk and fruit juices. The patient should rest. If the fever persists for three days, CBC should be performed to prevent deterioration of the condition, even if the dehydration is absent. Intravenous fluid should be administered to patients in whom lifesaving is required if administered at the right time (Oishi K, Saito M, Mapua CA, Natividad FF. 2007; 13: 125-33). 


\section{Health education and Prevention}

Health professionals are capable of providing extensive care program for many patients. Adequate training resources and expert advice are provided if necessary. Primary health care professionals and nurses can play an important role in hygiene and health education [Pal T, Dutta SK, Manda S, Saha B, Tripathi A. 2014; 61: 36570). Popular sources of information, such as television and newspapers, must be used to spread a wide range of information (Premaratna R, Bailey MS, Rtnasena BGN, de Silva HJ. 2007; 101: 683-5).

Protection against mosquito bites should be done using long-sleeved clothing and long clothing to cover the use of repellents, electric steam mats and mosquito coils throughout the day. In order to protect pregnant women, young children and the old persons, there are nets treated with insecticide (ITN), among others, which can be rested during the day. Water-containing objects such as tanks, drums, drums, buckets, air coolers should be emptied and the plants removed from the house when not in use. Stored water containers should be properly covered each time.

(Jardim DL, Tsukumo DM, Angerami RN, Carvalho Filho MA, Saad MJ. 2012; 16: 92-5) To prevent access by laying eggs from female mosquitoes, proper disposal of solid waste is encouraged and water storage practices are developed, including container coverage through community programs (Aneja VK, Kochar G, Bisht N. 2010; 7: 69).

Suggested measures to improve the situation.

To reduce the prevalence and number of disease-related deaths, the carrier should have early detection and control and improve the clinical management of DF. Currently, the most important element is not only the opinion of the community, but also for medical health professionals working in Pakistan, a good understanding of the disease, so that those who complain of symptoms Probably DF is properly managed. Standard care can be provided by health professionals following the development of a protocol. In order to do so, the Pakistani government needs to deploy personnel and mobilize strategies.

The population living in Pakistan faces great difficulties in accessing medical care and is becoming more difficult where the population is based in rural areas. A strategy should be made to ensure effective treatment in all areas. It is important to address the power and perspectives of social and general media, whereas the ability to absorb the maximum knowledge of the given information largely depends on the level of literacy in rural areas.

Literature mainly deals with the data of the big city population. In fact, this does not give us a real picture of the real burden of the disease. We can certainly hypothesize from the data provided, but an even more complete epidemiological study and the use of electronic patient registration systems will provide accurate information to help adequately address scarce resources. To address the DF outbreak, there should be strategies, such as nationalized forms with management plans, the deployment of equipment required for diagnosis and treatment at national level, and consistent recruitment and placement at times of outbreaks. In addition, resources should be allocated to monitor DF levels when there is no outbreak; Investigating and understanding outbreak patterns can also help direct resources and mobilize staff and team appropriately. The vector control is a signal, and the calculation must be performed with the closely monitoring to control the vector. The prevention strategies proposed by the World Health Organization (WHO) differ fundamentally in vector control. Although persistent or well-financed programs are difficult to implement, this will be the main suspension of preventive measures due to political and sectarian violence in Pakistan. Of course, water systems need to be improved to improve the trajectories and the welfare of the country, but this can be an important task. In addition to improving street cleanliness and better collection of household waste, there are other major problems. Immediate or simpler options may be, for example, to change the market coding to ensure that all water containers are sold with airtight closures.

\section{CONCLUSION}

Doctors play a vital role in the care, promotion and identification of the symptom of immediate bleeding, a serious consequence of dengue fever, and therefore refer to tertiary care to replace intravenous fluids, platelet transfusion and supportive care is required. Public awareness campaigns should start at all levels.

Doctors in professional health care institutions have the opportunity not only to offer their patients the best possible supportive care recommendations, but also to improve dengue and vector control spread. 


\section{References}

1.Malavige GN, Fernando S, Fernando DJ, Seneviratne SL. Dengue viral infections. Postgrad Med J 2004; 80:588-601.

2. Shaista Manzoor, Muhammad Afzal, Muhammad Hussain, Prof. Dr. Syed Amir Gilani Knowledge Attitude and Practice towards Dengue Fever Prevention among Adult Population of Rural Area of Lahore Pakistan International Journal of Scientific \& Engineering Research Volume 9, Issue 5, May-2018

3. Anita SB. Dengue fever, DHF and DSS. Apollo Med 2007; 4: 111-7

4. Keating J. An investigation into the cyclical incidence of dengue fever. Social Sci Med 2001; 53: 1587-97

5. Ilyas M, Rahman Z, Shamas S, Alam M, Israr M, M Masood. Bioinformatics Analysis of Envelope

Glycoprotein E epitopes of Dengue Virus Type 3. Afr J Biotechnol 2011; 10: 3528-33.

6. Firdous J, Nanji k, Qidwae W, Roshan R, Waseem H. Clinical and biochemical characteristics of suspected dengue fever in an ambulatory care family medical clinic, Aga Khan University, Karachi, Pakistan. Dengue Bull 2011; 35:59-64.

7. Durbin AP, Vargas MJ, Wanionek K, Hammond S, Gordon A, Rocha C, et al. Phenotyping of peripheral blood mononuclear cells during acute dengue illness demonstrates infection and increased activation of monocytes in severe cases compared to classic dengue fever. Virol 2008; 376: 329-35.

8. Kim JH, Chong CK, Sinniah M, Sinnadurai J, Song HO, Park H. Clinical diagnosis of early dengue infection by novel one-step multiplex real-time RT-PCR targeting NS1 gene. J Clin Virol 2015; 65: 11-9.

9. Zhang B, Salieb-Beugelaar GB, Nigo MM, Weidmann M, Hunziker P. Diagnosing dengue virus infection: rapid tests and the role of micro/nanotechnologies. Nanomedicine 2015; 11: 1745-61.

10. Guzman MG, Kouri G. Dengue and dengue hemorrhagic fever in the Americas: lessons and challenges. J ClinVirol 2003; 27: 1-13.

11. Kumar K, Singh PK, Tomar J, Baijal S. Dengue: epidemiology, prevention and pressing need for vaccine development. Asian Pacific J Trop Med 2010; 3: 997-1000.

12. Gibbons RV, Vaughn DW. Dengue: an escalating problem. BMJ 2002; 324: 1563-6

13. WHO. Managing Regional Public Goods for Health; Community-based Dengue Vector Control 2013; RPT125191-2.

14. Ooi EE, Goh KT, Gubler DJ. Dengue Prevention and 35 Years of Vector Control in Singapore. Emerg Infec Dis 2006; 12: 887-92.

15. Rigau-Pérez JG, Clark GG, Gubler DJ, Reiter P, Sanders EJ, Vorndam AV. Dengue and dengue haemorrhagic fever. Lancet 1998; 352: 971-7.

16. Wasay M, Channa R, Jumani M, Zafar A. Changing patterns and outcome of Dengue infection; report from a tertiary care hospital in Pakistan. J Pak Med Assoc 2008; 58:488-9.

17. Siddiqui FJ, Haider SR, Bhutta ZA. Endemic Dengue Fever: a seldom recognized hazard for Pakistani children. J Infect Dev Ctries 2009; 3: 306-12.

18. Rasheed SB, Butlin RK, Boots M. A review of dengue as an emerging disease in Pakistan. Public Health 2013; 127:11-7.

19. Khan E, Hasan R. Dengue infection in Asia; a regional concern. J Postgrad Med Inst 2011; 26: 1-

20. Hassan U, Loya A, Mehmood MT, Nazeer H, Sultan F. Dengue Fever Outbreak in Lahore. J Coll Physicians Surg Pak 2013; 23: 231-3.

21. Ashley EA. Dengue fever. Trends Anaesth Crit Care 2011; 1: 39-41.

22. Oishi K, Saito M, Mapua CA, Natividad FF. Dengue illness: clinical features and pathogenesis J Infect Chemother 2007; 13: 125-33.

23. Pal T, Dutta SK, Manda S, Saha B, Tripathi A. Differential clinical symptoms among acute phase Indian patientsrevealed significant association with dengue viral load and serum IFN-gamma level. J Clin Virol 2014; 61: 365-70.

24. Premaratna R, Bailey MS, Rtnasena BGN, de Silva HJ. Dengue fever mimicking acute appendicitis. Trans R Soc Trop Med Hyg 2007; 101: 683-5.

25. Jardim DL, Tsukumo DM, Angerami RN, Carvalho Filho MA, Saad MJ. Autoimmune features caused by dengue fever: a case report. Braz J Infec Dis 2012; 16: 92-5.

26. Aneja VK, Kochar G, Bisht N. Unusual manifestations of Dengue fever. Apollo Med 2010; 7 